\title{
Characteristics of Patients Meeting the New Definition of Pre-capillary Pulmonary Hypertension (Nice 2018) in a Single Japanese Pulmonary Hypertension Center
}

Keiko Yamamoto ( $\nabla$ shiitake@mx7.ttcn.ne.jp )

Chiba University

Nobuhiro Tanabe

Saiseikai Narashino Hospital

Yukiko Takahashi

Chiba University

Akira Naito

Chiba University

Ayumi Sekine

Chiba University

Rika Suda

Chiba University

Takayuki Jujo Sanada

Vrije Universiteit Medische Centrum

Toshihiko Sugiura

Chiba University

Ayako Shigeta

Chiba University

\section{Seiichiro Sakao}

Chiba University

Koichiro Tatsumi

Chiba University

\section{Research Article}

Keywords: pulmonary arterial hypertension, World Symposium on Pulmonary Hypertension (WSPH) 2018, pulmonary artery wedge pressure, pulmonary vascular resistance

Posted Date: March 18th, 2021 
DOl: https://doi.org/10.21203/rs.3.rs-295379/v1

License: (c) (1) This work is licensed under a Creative Commons Attribution 4.0 International License. Read Full License

Version of Record: A version of this preprint was published at BMC Pulmonary Medicine on August 9th, 2021. See the published version at https://doi.org/10.1186/s12890-021-01623-2. 


\section{Abstract}

Background: The $6^{\text {th }}$ World Symposium on Pulmonary Hypertension (Nice 2018) proposed a new definition of pre-capillary pulmonary hypertension $(\mathrm{PH})$ as a condition with mean pulmonary artery pressure $(\mathrm{mPAP})>20 \mathrm{mmHg}$, pulmonary artery wedge pressure $(\mathrm{PAWP}) \leq 15 \mathrm{mmHg}$, and pulmonary vascular resistance (PVR) $\geq 3$ Wood units (WU). The characteristics and prognosis of patients with precapillary $\mathrm{PH}$, according to this new definition, is unclear. Therefore, we determined the characteristics of patients with pre-capillary $\mathrm{PH}$ who had pulmonary vascular disease with slight elevation in mPAP.

Methods: We retrospectively enrolled 683 patients who underwent their first right heart catheterization $(\mathrm{RHC})$ at Chiba University, Japan. Among them, 489 patients met the pre-capillary $\mathrm{PH}$ requirement with $\mathrm{mPAP} \geq 25 \mathrm{mmHg}$ (conventional pre-capillary PH group), while 22 patients met the borderline precapillary $\mathrm{PH}$ criteria (borderline pre-capillary $\mathrm{PH}$ group).

Results: The borderline pre-capillary PH group comprised $4.3 \%$ of the total pre-capillary PH patients, and the majority was in Group 3 (40.9\%) or 4 (45.5\%). The survival of the borderline pre-capillary PH group tended to be better than that of the conventional pre-capillary $\mathrm{PH}$ group.

Conclusions: Pre-capillary PH patients, according to the Nice 2018 definition, comprised $4.3 \%$ of the population with total pre-capillary $\mathrm{PH}$ and had better survival than the conventional $\mathrm{PH}$ pre-capillary group; moreover, the majority of the pre-capillary PH cases were either Group 3 and 4.

\section{Background}

The World Symposium on Pulmonary Hypertension (WSPH) considered the scientific and clinical knowledge concerning pulmonary hypertension $(\mathrm{PH})$ and proposed a new definition of $\mathrm{PH}$ and new treatment strategies. The general purpose of the clinical classification of $\mathrm{PH}$ is to categorize clinical conditions associated with $\mathrm{PH}$ based on similar pathophysiological mechanisms, clinical presentation, hemodynamic characteristics, and therapeutic management. A comprehensive and simplified updated version of the clinical classification of PH was presented in Nice 2018 [1]. To date, since the first WSPH in 1973, PH has been arbitrarily defined as mean pulmonary arterial pressure (mPAP) $\geq 25 \mathrm{mmHg}$ at rest. However, recent data obtained for patients undergoing right heart catheterization (RHC) have shown that normal mPAP was $14.0 \pm 3.3 \mathrm{mmHg}$ in healthy subjects, and two standard deviations above this mean value would suggest that mPAP $>20 \mathrm{mmHg}$ is above the upper normal limit [1, 2]. In addition, the mPAP value is not sufficient to define pulmonary vascular disease because this value can be affected by the cardiac output (CO) or pulmonary arterial wedge pressure (PAWP). Considering these aspects, the task force of the $6^{\text {th }}$ WSPH in 2018 proposed that if all three criteria are met, namely, mPAP $>20 \mathrm{mmHg}$, PAWP $\leq 15 \mathrm{mmHg}$, and pulmonary vascular resistance (PVR) $\geq 3$ Wood units (WU), then the new definition of pre-capillary PH would be satisfied [1]. A PAWP $>15 \mathrm{mmHg}$ is defined as post-capillary $\mathrm{PH}$, which is considered as isolated PH when PVR $<3 \mathrm{WU}$, and combined pre- and post-capillary PH when PVR $\geq 3 W U$. In terms of pulmonary vascular disease, the reliability and validity of this new $\mathrm{PH}$ definition have not been 
defined. In addition, the characteristics of patients with pre-capillary $\mathrm{PH}$, diagnosed according to this new definition, are unclear. Therefore, in our cohort of patients with $\mathrm{PH}$, we determined the characteristics of patients with pre-capillary $\mathrm{PH}$ who had pulmonary vascular disease with a modest elevation in mPAP (borderline pre-capillary PH group).

\section{Methods}

\section{Ethics approval and consent to participate}

Patient identity was concealed, and all data were compiled according to the requirements of the Japanese Ministry of Health, Labour and Welfare, which is dedicated to privacy, information technology, and civil rights. Based on the Japanese legislation, the need for informed consent was waived. The study protocol was approved by the Research Ethics Committee of Chiba University School of Medicine (approval number: 2,584). Since 2009, all survivors have provided written informed consent for a prospective cohort study (approval number 826 ). For patients who died before 2008, written informed consent was not required, in line with the guidelines for retrospective studies in Japan and in accordance with the criteria of the ethics committee of Chiba University Hospital. The study database was anonymized and all experiments were performed in accordance with the relevant guidelines and regulations.

\section{Patients}

Our patients were mainly referred from other hospitals or other departments of Chiba University Hospital. Our $\mathrm{PH}$ center is associated with respiratory medicine. Therefore, patients with respiratory diseases suspected of $\mathrm{PH}$ during follow-up were also included. Among them, we conducted a retrospective analysis of data from patients who had undergone RHC. Indication criteria for RHC were as follows: RHC was conducted when a patient's hypoxia or exercise limitation was considered to have stemmed from $\mathrm{PH}$, and the outcome of RHC seemed to have influenced the patient's treatment, including their eligibility for clinical trials. Especially, among patients with respiratory diseases, $\mathrm{RHC}$ was conducted if transplantation was indicated or when RHC would be beneficial. Among the 1,542 patients who underwent RHC from 1999 to 2020 at Chiba University, we enrolled 683 cases who were catheterized for the first time at the first diagnosis. The reason for choosing patients from 1999 was that in Japan, epoprostenol was approved in 1999, and since then, patients could be prescribed selective pulmonary vasodilators. Of these, 531 patients had mPAP $\geq 25 \mathrm{mmHg}$ (conventional $\mathrm{PH}$ group) and 50 patients had mPAP ranging from 20 to $24 \mathrm{mmHg}$ (borderline PH group). The non-PH group consisted of 102 patients with mPAP < 20 $\mathrm{mmHg}$ (Figure 1a). We then chose PH patients with pulmonary vascular disease who met the criteria for pre-capillary PH (mPAP $>20 \mathrm{mmHg}$ with PAWP $\leq 15 \mathrm{mmHg}$ and $\mathrm{PVR} \geq 3 \mathrm{WU}$ ). Among the pre-capillary PH patients ( $n=511)$, mPAP $\geq 25 \mathrm{mmHg}$ was found in 489 (conventional pre-capillary PH group) and mPAP 25>mPAP>20 mmHg was found in 22 patients (borderline pre-capillary PH group) (Figure 1b). We also categorized our cohort into Group 1-5 according to the Nice 2018 classification[1] . Occasionally, classifying Classes 1 and 3 was difficult, despite following the new Nice 2018 recommendation, 
especially when judging morphological or physiological severity. In this study, we allocated patients to Group1 to 5 after evaluation by two pulmonologists.

For Study 1 we compared the characteristics of the conventional $\mathrm{PH}$, borderline $\mathrm{PH}$, and non-PH groups. The non-PH group was used as a reference because the main aim was to clarify the characteristics of patients diagnosed with $\mathrm{PH}$ based on the new definition of pre-capillary $\mathrm{PH}$.

For Study 2, to focus on the pre-capillary nature of $\mathrm{PH}$, characteristics of the conventional pre-capillary $\mathrm{PH}$ and borderline pre-capillary PH groups were compared.

With respect to survival, all-cause mortality or lung transplantation was determined in all subjects. We excluded patients with a history of pulmonary endarterectomy (PEA) because it has been established that PEA improves the prognosis of patients with chronic thromboembolic pulmonary hypertension (CTEPH) [3].

\section{Statistical analysis}

To evaluate the baseline characteristics in each group, we used Student's t-tests to compare continuous variables and chi-square tests to compare categorical variables. Differences among three groups were evaluated using a one-way analysis of variance (ANOVA). The results are displayed as mean \pm standard deviation or median (interquartile range) for continuous variables, and the number (\%) for categorical variables. Survival curves were determined using the Kaplan-Meier method and the values were compared with the Wilcoxon test. A p-value $<0.05$ was considered significant. All analyses were performed using the JMP Pro software 13.2.0, Japanese version (SAS Institute Inc).

\section{Results}

1. Conventional PH and borderline PH group (Study 1)

Among the patients who underwent RHC for the first time $(n=683)$, the number of patients in the conventional PH group was 531 (77.7\%), and that in the borderline PH group was 50 (8.6\% of total PH patients) (Figure 1a).

The majority of the borderline PH patients were in Group 3 (30\%) and Group 4 (52\%) (Table 1). However, according to the clinical classification, the majority of borderline $\mathrm{PH}$ patients among the total patients in their specific Groups, was still in Group 3 (15\%), and only 6.8\% were in Group 4 (Table 1).

Detailed analysis, based on etiology, showed that hemodynamics, alveolar-arterial oxygen difference $\left(\mathrm{AaDO}_{2}\right)$, gas exchange impairment, and 6-minute walk distance (6MWD) were worst in the conventional group (Table 2). In addition, the partial pressure of arterial carbon dioxide $\left(\mathrm{PaCO}_{2}\right)$ was the lowest in the conventional PH group. In Group 3, no significant differences in parameters of the ventilatory function were observed among the conventional, borderline $\mathrm{PH}$, and non- $\mathrm{PH}$ groups. 
Next, we compared the survival of the conventional PH group, borderline group, and non-PH group (Figure 2). The survival of the conventional PH group was worse than that of the borderline group, and it was the worst among all three groups. Analogical tendencies were found in Group 1 and 4. However, in Group 3, the 10 year survival of all groups was less than $40 \%$. Group 3 showed poor prognosis, even in the non-PH group. Additionally, we divided patients of Group 3 into two sub-groups according to the time of diagnosis (diagnosed in 1999-2009 or 2010-2020). The ratio of non-PH group diagnosed in 1999-2009 was higher than that diagnosed in 2010 (Table 3-a). Moreover, we analyzed the cause of death among Group 3 patients. The number of patients who died due to malignant disease and who underwent lung transplantation was higher in the non-PH group than in the $\mathrm{PH}$ group (Table 3-b).

\section{Characteristics and survival of pre-capillary PH patients (Study 2)}

The number of patients in the conventional pre-capillary PH group was 489 (71.6\%). Twenty-two patients (3.2\% of the total patients, including the non-PH patients; $4.3 \%$ of the total pre-capillary PH patients) were included in the borderline pre-capillary PH group (Figure $1 \mathrm{~b}$ and Table 4). Similar to Study 1 , the majority of the borderline pre-capillary PH patients belonged to Group 3 (40.9\%) and 4 (45.5\%). However, in the percentage of borderline pre-capillary $\mathrm{PH}$ patients among the total pre-capillary $\mathrm{PH}$ patients, the majority belonged to Group 3 (13.2\%) and only 3.2\% patients belonged to Group 4 (Table 4).

Regarding baseline characteristics, in addition to hemodynamics, partial pressure of arterial oxygen $\left(\mathrm{PaO}_{2}\right)$, partial pressure of mixed venous oxygen $\left(\mathrm{PvO}_{2}\right)$, and $\mathrm{AaDO}_{2}$ in the conventional pre-capillary $\mathrm{PH}$ group were significantly worse than those in the borderline pre-capillary $\mathrm{PH}$ group. In addition, $\mathrm{PaCO}_{2}$ was significantly lower in the conventional pre-capillary $\mathrm{PH}$ group. There was no significant difference in the ventilatory function between the two groups.

Focusing on each etiological group, the conventional pre-capillary $\mathrm{PH}$ group showed more severe hemodynamics and gas exchange impairment than the borderline pre-capillary PH group in Group 1, 3, and 4. A significantly lower $\mathrm{PaCO}_{2}$ in the conventional pre-capillary $\mathrm{PH}$ group was found only in Group 1 relative to that in the other Groups. Even in Group 3, there was no significant difference in parameters of ventilatory function between the two groups (Table 5).

Furthermore, the survival of the conventional pre-capillary $\mathrm{PH}$ group was worse than that of the borderline pre-capillary PH group, but it did not reach significance. The same tendency was observed in Group 1, 3 , and 4. In Group 3, the 5- and 10-year survival rates in the conventional pre-capillary PH population were $39.8 \%$ and $22.4 \%$, respectively. Those in the borderline pre-capillary $\mathrm{PH}$ group were $100 \%$ and $50 \%$, respectively (Figure 3).

\section{Discussion}

This study revealed the characteristics and survival of patients diagnosed with pre-capillary $\mathrm{PH}$, according to new diagnostic criteria proposed at the WSPH 2018. 
The borderline pre-capillary PH group accounted for $3.2 \%$ (22 in 683) of the total patient population who underwent their first RHC and $4.3 \%$ of all pre-capillary PH patients (22 in 511). The borderline PH group was $8.6 \%$ (50 in 581) of total $\mathrm{PH}$ patients. The majority of the borderline pre-capillary $\mathrm{PH}$ group belonged to Group 3 and 4.

Most of the previous studies focused on patients with mPAP 25>mPAP>20 $\mathrm{mmHg}$, the so-called "borderline $\mathrm{PH}$," and did not focus on the pre-capillary nature. The percentage of borderline $\mathrm{PH}$ patients was $4.2-18 \%$ among all patients and $4.5-22.6 \%$ of all patients with mPAP $>20 \mathrm{mmHg}[4],[5],[6]$. Assad et al. showed that the percentage of patients with mPAP between 19 and $24 \mathrm{mmHg}$ among all patients, including Non-PH patients (20.1\%), was $18 \%$ and that the majority of them belonged to Group 2 because over $70 \%$ patients had cardiovascular disease [4]. Douschan et al. found that $11.7 \%$ of all patients, including those without $\mathrm{PH}(35.2 \%)$, had borderline $\mathrm{PH}[6]$. In their study, $20.3 \%$ of patients belonged to Group 2 with overt $\mathrm{PH}$, and patients with borderline $\mathrm{PH}$ and patients with overt $\mathrm{PH}$ showed a higher risk of cardiac disease. These results suggest that the main background factor of these patients was cardiac disease. However, in these studies detailed demographic data, including number of patients without $\mathrm{PH}$, were not known [4],[5],[6]. Another study showed that the total percentage of borderline PH patients was only $4.2 \%$ in total, including Non-PH patients (5.7\%) [6]. In that study, among borderline PH and patients without $\mathrm{PH}$, a relatively lower percentage of left heart disease $(16.2 \%)$ and a relatively higher percentage of respiratory disease $(29.7 \%)$ were found. However, the background status of all patients, including those with overt $\mathrm{PH}$, is not known (Table 6).

In our study, the number of borderline PH group patients, including PH Group 1 to 5 , was $8.6 \%$, lower than that reported in Assad's and Douschan's studies (22.6\% and 18.1\%, respectively) (Table 1 ), and the majority of the total patients belonged to Group $3(14.6 \%)$ and $4(55.6 \%)$. We can explain the relatively higher ratio of these groups since our $\mathrm{PH}$ center is associated with respiratory medicine and is one of the high-volume PEA centers in Japan. In our study, the ratio of borderline PH is relatively high in Group 3. Similarly, a study of severe COPD patients who underwent lung transplantation also showed that the majority had a mild elevation of mPAP (20-25 mmHg) [7]; therefore, the majority of patients with severe lung disease tended to have a mild elevation of mPAP. On the other hand, in Group 4, our data showed that the ratio of borderline $\mathrm{PH}$ was low. The Papworth hospital study, which is another PEA center, reported chronic thromboembolic disease with mPAP $<25 \mathrm{mmHg}$ in only 42 of 1019 patients (4.1\%) who underwent PEA [8].

The higher percentage of Group 4 patients in our center may explain the lower percentage of the borderline $\mathrm{PH}$ group in total. Overall, the number of patients who met the new diagnostic criteria depended on their background status.

Regarding baseline characteristics, in addition to hemodynamics, the $\mathrm{PaO}_{2}, \mathrm{PvO}_{2}$, and $\mathrm{AaDO}_{2}$ were better in the borderline pre-capillary $\mathrm{PH}$ group than in the conventional pre-capillary $\mathrm{PH}$ group. Lower $\mathrm{PaCO}_{2}$ in Group 1 of the conventional PH group could suggest the hyperventilation is compensating for gas exchange impairment. 
Several studies have shown little correlation between ventilatory function and severity of PH in patients with lung disease [9],[10],[11]. Similarly, in our study, there was no significant difference in ventilatory function between the conventional and the borderline pre-capillary PH groups in Group 3.

In Study 1 , the survival of the conventional PH group was worse than that of the borderline PH group. Similarly, in Study 2, the survival of the conventional pre-capillary PH group was worse than that of the borderline pre-capillary PH group, though no significant difference between the borderline PH group and the non-PH group was observed.

Previous data suggested that mild elevation of $\mathrm{PH}$ is associated with poor prognosis in idiopathic pulmonary fibrosis [12] or chronic obstructive pulmonary disease [13],[14]. Assad et al. also showed poor prognosis in patients with borderline $\mathrm{PH}$, and the majority of patients seemed to be in Group 2 [4]. Douchan et al. revealed poorer prognosis and increased cardiopulmonary comorbidities in patients with mPAP of $17-26 \mathrm{mmHg}$ than for those patients with mPAP $<17 \mathrm{mmHg}$ [5]. They chose patients having similar background status in both the $\mathrm{PH}$ and non-PH groups. Although a report including patients with relatively heterogeneous background diseases also showed poor prognosis of borderline $\mathrm{PH}$ patients, the difference in prognosis between overt $\mathrm{PH}$ and borderline $\mathrm{PH}$ patients was detected when they focused on patients in Group 1 [6]. In our study, Group 3 cases had poor prognosis, even in the non-PH group. Additional analysis showed that the number of Non-PH patients was higher from 1999-2009 than that from 2010-2020. This means that Non-PH patients may not have received better treatment than those diagnosed in 2010-2020. In addition, the number of patients who died of malignant disease and underwent lung transplantation was higher in the non-PH group. These underlying conditions may have affected the poor prognosis of the non-PH group in Group 3. The prognosis of patients with slightly increased mPAP could not only be determined by their hemodynamics but also by patients' background conditions. Accordingly, prospective studies are needed to evaluate whether such patients should be prescribed vasodilators.

\section{Limitations}

This was a retrospective single-center study, and the sample size was relatively small to evaluate the precapillary PH group effectively. Furthermore, our PH center specializes in respiratory medicine. This may have affected the patient cohort. In Group 3, we could not examine extensive lung disease by computed tomography. In the present study, follow-up data after any treatment intervention was not analyzed because there were insufficient data to evaluate the change in patient status.

\section{Conclusions}

Patients who were included in the pre-capillary PH group according to the Nice 2018 definition accounted for $4.3 \%$ of the pre-capillary PH patients, and the majority of them were in Group 3 and 4 . Further prospective studies are needed to determine whether we should prescribe $\mathrm{PH}$-specific treatment or not. 


\section{Abbreviations}

WSPH, The World Symposium on Pulmonary Hypertension

$\mathrm{PH}$, pulmonary hypertension

mPAP, mean pulmonary arterial pressure

$\mathrm{RHC}$, right heart catheterization

CO, cardiac output

PAWP, pulmonary arterial wedge pressure

PVR, pulmonary vascular resistance

PEA, pulmonary endarterectomy

CTEPH, chronic thromboembolic pulmonary hypertension

ANOVA, one-way analysis of variance

$\mathrm{AaDO}_{2}$, alveolar-arterial oxygen difference

6MWD, 6-minute walk distance

$\mathrm{PaCO}_{2}$, partial pressure of arterial carbon dioxide

$\mathrm{PaO}_{2}$, partial pressure of arterial oxygen

$\mathrm{PvO}_{2}$, partial pressure of mixed venous oxygen

\section{Declarations}

\section{Acknowledgments}

This study was supported in part by grants from the Intractable Respiratory Diseases and Pulmonary Hypertension Research Group, the Ministry of Health, Labor and Welfare, Japan (H29-027), and the Pulmonary Hypertension Research Group of the Japan Agency for Medical Research and Development, AMED (17ek0019127h0003).

\section{Ethics approval and consent to participate}

We protected the identity of patients' compiled data according to the requirements of the Japanese Ministry of Health, Labor, and Welfare, dedicated to privacy, information technology, and civil rights. The Research Ethics Committee of Chiba University School of Medicine approved the study's protocol 
(approval number 2,584). Since 2009, all survivors provided written informed consent for a prospective cohort study (approval number 826). Deaths before 2008 were not required to provide written informed consent as per the guidelines for retrospective studies in Japan and the ethics committee of Chiba University Hospital permit it (Study 2). The study database was anonymized. All experiments were performed in accordance with the relevant guidelines and regulations.

\section{Consent for publication}

Not applicable

\section{Availability of data and materials}

The study database was anonymized, and the study complied with the requirements of the Japanese Ministry of Health, Labour and Welfare. The datasets analyzed during the current study are not publicly available, but are available from the corresponding author on a reasonable request and with the permission of our department.

\section{Conflict of interest}

The authors have no conflicts of interest to declare for this article.

\section{Funding}

Not applicable

\section{Authors' contributions}

$\mathrm{KY}, \mathrm{NT}$, and KT wrote the main manuscript text. KY and YT analyzed the data. NT, YT, AN,AS,RS,TJS,TS,AS,SS, and KT reviewed the manuscript. All authors read and approved the final manuscript.

\section{References}

1. Simonneau G, Montani D, Celermajer DS, Denton CP, Gatzoulis MA, Krowka M, Williams PG, Souza R: Haemodynamic definitions and updated clinical classification of pulmonary hypertension. Eur Respir J 2019, 53(1).

2. Kovacs G, Berghold A, Scheidl S, Olschewski H: Pulmonary arterial pressure during rest and exercise in healthy subjects: a systematic review. Eur Respir J 2009, 34(4):888-894.

3. Jenkins D, Madani M, Fadel E, D'Armini AM, Mayer E: Pulmonary endarterectomy in the management of chronic thromboembolic pulmonary hypertension. Eur Respir Rev 2017, 26(143).

4. Assad TR, Maron BA, Robbins IM, Xu M, Huang S, Harrell FE, Farber-Eger EH, Wells QS, Choudhary G, Hemnes AR et al: Prognostic Effect and Longitudinal Hemodynamic Assessment of Borderline Pulmonary Hypertension. JAMA Cardio/ 2017, 2(12):1361-1368. 
5. Douschan P, Kovacs G, Avian A, Foris V, Gruber F, Olschewski A, Olschewski H: Mild Elevation of Pulmonary Arterial Pressure as a Predictor of Mortality. Am J Respir Crit Care Med 2018, 197(4):509-516.

6. Heresi GA, Minai OA, Tonelli AR, Hammel JP, Farha S, Parambil JG, Dweik RA: Clinical characterization and survival of patients with borderline elevation in pulmonary artery pressure. Pulm Circ 2013, 3(4):916-925.

7. Andersen KH, Iversen M, Kjaergaard J, Mortensen J, Nielsen-Kudsk JE, Bendstrup E, Videbaek R, Carlsen J: Prevalence, predictors, and survival in pulmonary hypertension related to end-stage chronic obstructive pulmonary disease. The Journal of heart and lung transplantation: the official publication of the International Society for Heart Transplantation 2012, 31(4):373-380.

8. Taboada D, Pepke-Zaba J, Jenkins DP, Berman M, Treacy CM, Cannon JE, Toshner M, Dunning JJ, Ng C, Tsui SS et al: Outcome of pulmonary endarterectomy in symptomatic chronic thromboembolic disease. Eur Respir J 2014, 44(6):1635-1645.

9. Nathan SD, Shlobin OA, Ahmad S, Urbanek S, Barnett SD: Pulmonary hypertension and pulmonary function testing in idiopathic pulmonary fibrosis. Chest 2007, 131(3):657-663.

10. Low AT, Medford AR, Millar AB, Tulloh RM: Lung function in pulmonary hypertension. Respir Med 2015, 109(10):1244-1249.

11. Hurdman J, Condliffe R, Elliot CA, Swift A, Rajaram S, Davies C, Hill C, Hamilton N, Armstrong IJ, Billings $C$ et al: Pulmonary hypertension in COPD: results from the ASPIRE registry. Eur Respir J 2013, 41(6):1292-1301.

12. Hamada K, Nagai S, Tanaka S, Handa T, Shigematsu M, Nagao T, Mishima M, Kitaichi M, Izumi T: Significance of pulmonary arterial pressure and diffusion capacity of the lung as prognosticator in patients with idiopathic pulmonary fibrosis. Chest 2007, 131(3):650-656.

13. Kessler R, Faller M, Fourgaut G, Mennecier B, Weitzenblum E: Predictive factors of hospitalization for acute exacerbation in a series of 64 patients with chronic obstructive pulmonary disease. $A m \mathrm{~J}$ Respir Crit Care Med 1999, 159(1):158-164.

14. Oswald-Mammosser M, Weitzenblum E, Quoix E, Moser G, Chaouat A, Charpentier C, Kessler R: Prognostic factors in COPD patients receiving long-term oxygen therapy. Importance of pulmonary artery pressure. Chest 1995, 107(5):1193-1198.

\section{Figures}




\section{Studv 1}

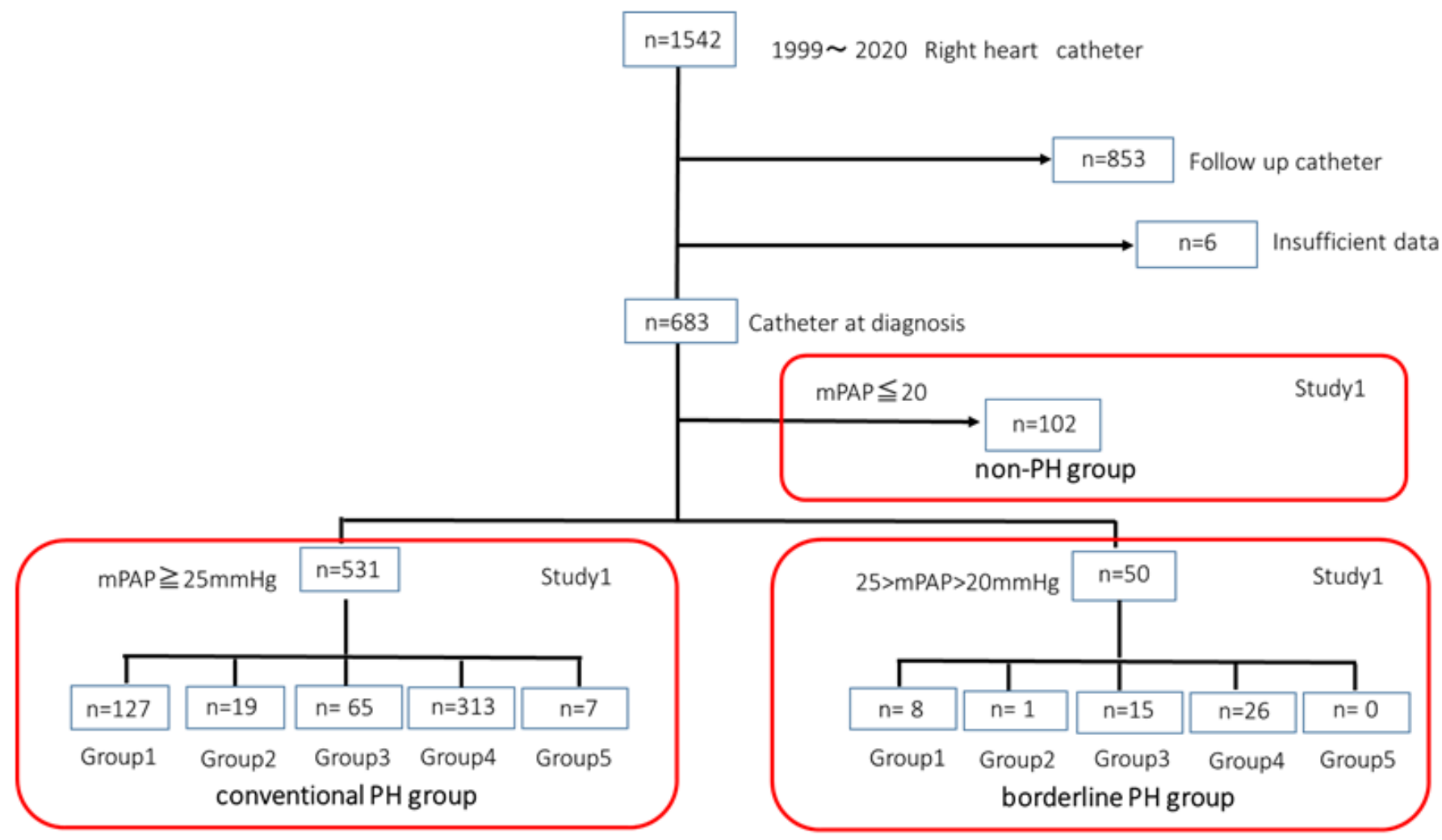

b

Study2

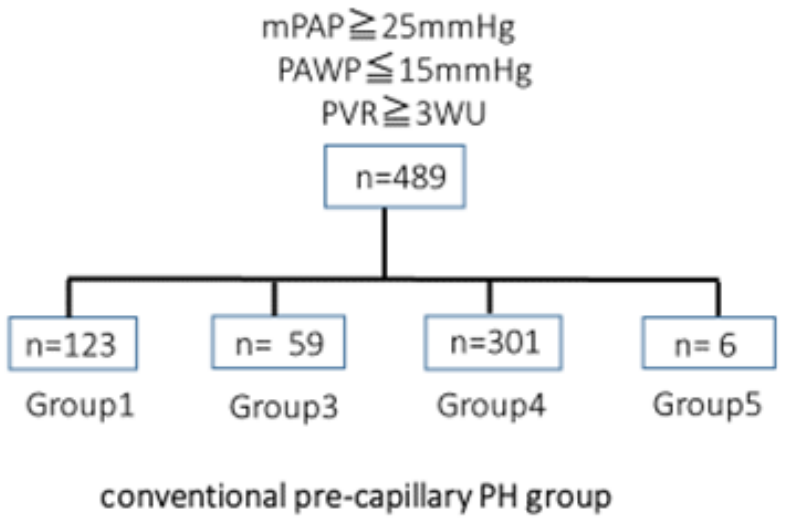

Figure 1

Selection of patients a. Among 1542 cases of right heart catheterization (RHC) handled at the Chiba University, Japan, we enrolled 683 patients who underwent the first RHC. Of these, 531 patients had $\mathrm{mPAP} \geq 25 \mathrm{mmHg}$ (the conventional PH group) and 50 patients had mPAP $25>\mathrm{mPAP}>20 \mathrm{mmHg}$ (the borderline $\mathrm{PH}$ group). There were 102 patients with $\mathrm{mPAP}<20 \mathrm{mmHg}$ (the non-PH group). b. We then chose $\mathrm{PH}$ patients with pulmonary vascular disease, namely pre-capillary $\mathrm{PH}$ patients (mPAP > 20 with 
PAWP $\leq 15 \mathrm{mmHg}$ and PVR $\geq 3 W U$ ). Among the pre-capillary PH patients $(n=501)$, in $489 \mathrm{mPAP}$ was $\geq$ $25 \mathrm{mmHg}$ (the conventional pre-capillary group) and 22 patients had mPAP $25>\mathrm{mPAP}>20 \mathrm{mmHg}$ (the borderline pre-capillary group).

Total
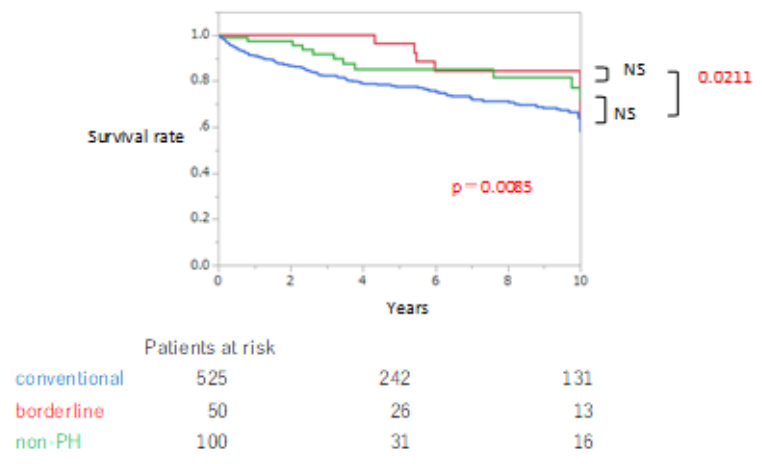

Group1

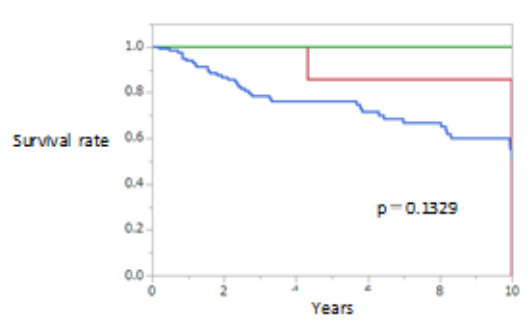

Patients at risk

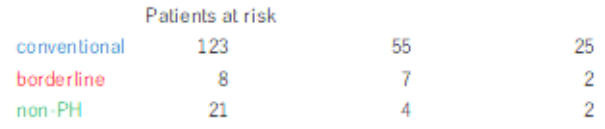

Group3

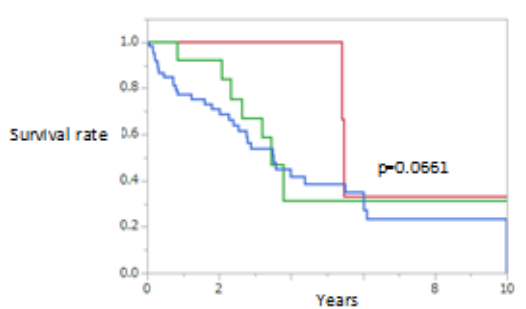

Patients at risk

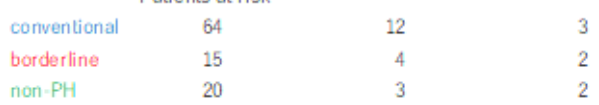

Group4

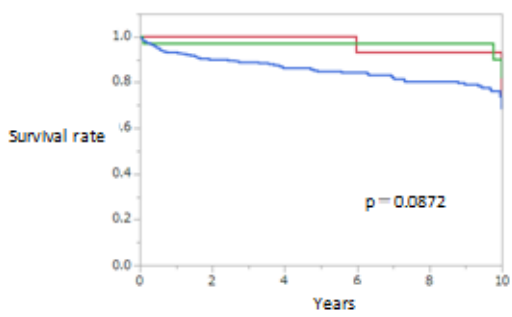

Patients at risk

$\begin{array}{lrrr}\text { conventional } & 312 & 170 & 100 \\ \text { borderline } & 26 & 17 & 11 \\ \text { non }-\mathrm{PH} & 39 & 21 & 12\end{array}$

Figure 2

Survival (Study 1) The survival of the conventional PH group was the worst among the 3 groups $(p=0.0085)$. There was no significant difference in the survival between the borderline group and the non- 
PH group.
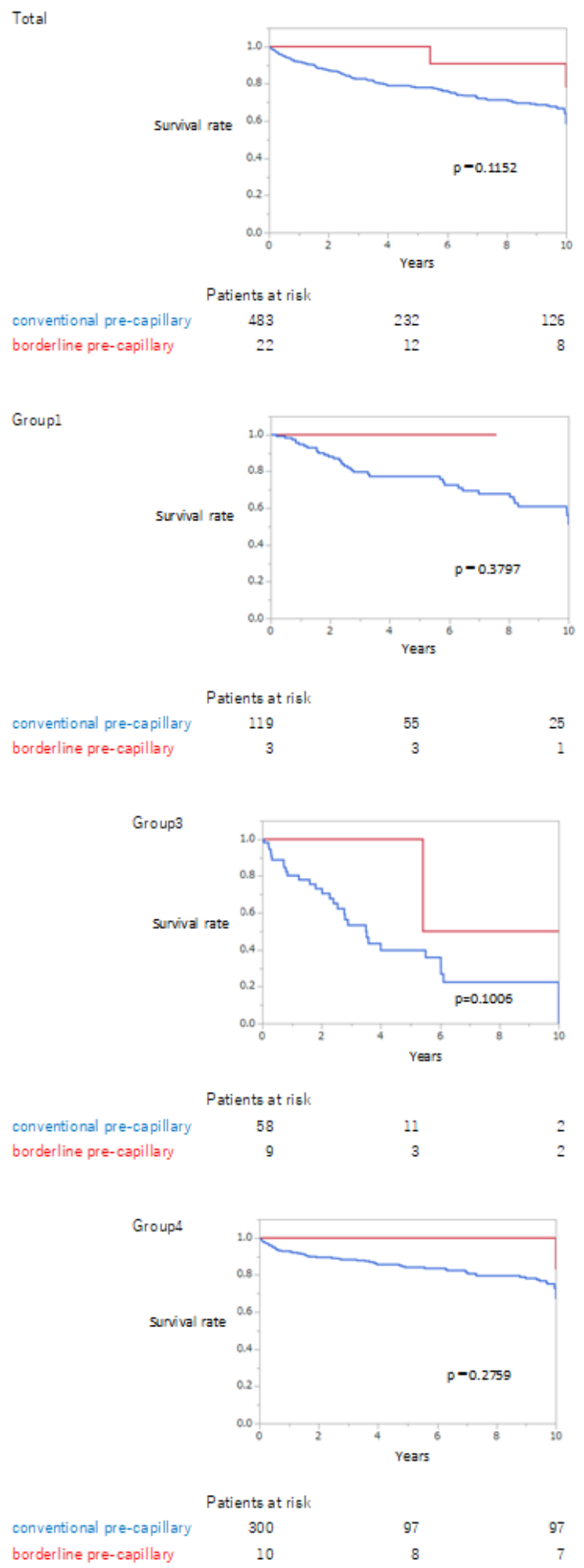

\section{Figure 3}

Survival (Study 2: the pre-capillary $\mathrm{PH}$ ) The survival of the conventional pre-capillary PH group was worse than that of the borderline pre-capillary PH group, but the difference was not significant. The same tendency was observed in Group 1, 3 and 4. In Group 3, the 5-year and 10-year survival rates in the 
conventional pre-capillary $\mathrm{PH}$ population were $39.8 \%$ and $22.4 \%$, respectively. The survival rates in the borderline pre-capillary $\mathrm{PH}$ group were $100 \%$ and $50 \%$, respectively. 